\title{
Sustentabilidad ambiental de las concesiones forestales en el departamento Huánuco, Perú
}

\section{Environmental sustainability of forestal concessions in Huanuco department, Peru}

\author{
Luis Alberto Valdivia Espinozal*, Tito Felipe Gonzalez Manrique de Lara² y Alberto Marcial Julca-Otiniano ${ }^{3}$
}

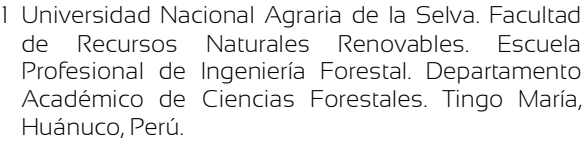

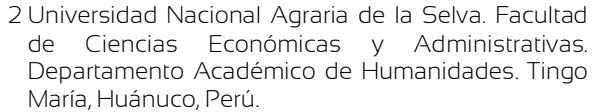

\author{
3 Universidad Nacional Agraria La Molina. Facultad de \\ Agronomía. Departamento Académico de \\ Fitotecnia. Lima, Perú. \\ * Autor de correspondencia. 20161490@lamolina.edu.pe
}

\section{RESUMEN}

$\mathrm{El}$ aprovechamiento sustentable de los recursos forestales consiste en utilizarlos para generar riqueza, mejorando indicadores sociales y evitando el desequilibrio ambiental. El objetivo del estudio fue determinar la sustentabilidad ambiental de las concesiones forestales en el departamento Huánuco, Perú, mediante el análisis multicriterio basado en el uso de indicadores. Se construyeron indicadores que incluyen la diversidad de macrofauna edáfica evaluada hasta $30 \mathrm{~cm}$ de profundidad, como evidencia del estado de conservación y mantenimiento del suelo. Los indicadores fueron estandarizados mediante escala de 0 a 4.0, siendo 4.0 el mayor valor de sustentabilidad; posteriormente, fueron ponderados multiplicando la escala por un coeficiente, según su importancia respecto a la sustentabilidad. Una concesión forestal es ambientalmente sustentable, si el índice de sustentabilidad ambiental (ISA) es $\geq 2.0$. La encuesta elaborada se aplicó a titulares y trabajadores de las concesiones forestales. Para conocer si el ISA en $60 \%$ de concesiones forestales supera el valor 2.0 , se empleó la prueba estadística no paramétrica chi cuadrada; también se realizó entre indicadores, la correlación de Pearson con un 95\% de confiabilidad. La prueba chi cuadrada confirma que las concesiones forestales con aprovechamiento forestal manual $(60 \%$ del total), son ambientalmente sustentables $\left(\mathrm{X}^{2}=0.056, \mathrm{p}\right.$-valor $\left.=0.814\right)$. Se registró fuerte correlación positiva estadísticamente significativa $(\mathrm{r}=0.970$, p-valor < 0.05) entre los indicadores "conservación y mantenimiento del recurso suelo" y "conservación de flora silvestre". El aprovechamiento forestal manual mantiene armonía con el entorno natural, y aunque el aprovechamiento forestal mecanizado sea de impacto reducido, genera daños al ecosistema.

PALABRAS CLAVE: análisis multicriterio, aprovechamiento forestal manual, aprovechamiento forestal mecanizado, impacto reducido, indicadores, índice.

\section{ABSTRACT}

The sustainable utilization of forest resources consists of using them to generate wealth, improving social indicators and avoiding environmental imbalance. The objective of the study was to determine the environmental sustainability of forest concessions in the Department of Huanuco, Peru, through a multicriteria analysis based on the use of indicators. Indicators were built including the diversity of edaphic macro-fauna evaluated down to $30 \mathrm{~cm}$ deep, as evidence of soil conservation and maintenance state. The indicators were standardized using a scale from 0 to 4.0, 4.0 being the highest value of sustainability; they were subsequently weighted by multiplying the scale by a coefficient, based on their importance with respect to sustainability. A forest concession is environmentally sustainable, if the Environmental Sustainability Index (ESI) is $\geq 2.0$. The designed survey was applied to stakeholders and workers of forestal concessions. To know if the ESI in $60 \%$ of forest concessions exceeds the value 2.0, the Chi Square test was used; Pearson's correlation was also performed between indicators, with $95 \%$ reliability. The Chi Square test confirms that forest concessions with manual forestal utilization $\left(60 \%\right.$ of the total) are environmentally sustainable $\left(\mathrm{X}^{2}=0.056\right.$, $\mathrm{p}$-value $\left.=0.814\right)$. A strong statistically significant positive correlation was recorded $(r=0.970$, p-value $<0.05)$ between "conservation and maintenance of soil resource" $(\mathrm{CMSR})$ and "conservation of wild flora" (CWFl) indicators. Manual forest utilization maintains harmony with the natural environment, and even when mechanized forestal is utilized with a reduced impact, it generates damage to the ecosystem.

KEYWORDS: multicriteria analysis, manual forest harvesting, mechanized forest harvesting, reduced impact, indicators, index. 


\section{INTRODUCCIÓN}

La primera definición oficial del concepto de desarrollo sustentable surge de un informe realizado en 1987 por la Comisión Brundtland (World Commission on Environment and Development, 1987), que considera que el desarrollo sustentable es "aquel que permite satisfacer las necesidades de las generaciones presentes sin comprometer las posibilidades de satisfacer las necesidades de las generaciones futuras", lo cual marcó el inicio para que la humanidad tomara conciencia de que el planeta Tierra constituye un espacio finito.

En el manejo forestal, uno de los términos más usados es el de "manejo sostenido", el cual hace referencia a la producción constante de madera; sin embargo, este concepto fue expandiéndose gradualmente hasta incluir la producción sostenida de otros productos forestales como agua, recreación, pesca, hábitat de fauna silvestre, forraje para ganado y otros no maderables (Floyd et al., 2001). Por esta razón, muchos autores usan como sinónimos "rendimiento sostenido" y "sustentabilidad forestal"; no obstante, Floyd y colaboradores sostienen que se trata de términos distintos, y que una de las diferencias más importantes es que el término "sustentabilidad forestal", además de la producción sostenida de los recursos forestales, hace énfasis en los procesos y funciones del ecosistema (purificación del aire y agua, formación del suelo, ciclo de energía y nutrientes, etc.).

Narváez et al. (2003) refieren al respecto que, actualmente, la mayor parte de los países del mundo han adoptado además del concepto de desarrollo sustentable, los términos "sustentabilidad forestal" y "manejo forestal sustentable"; ambos consideran el uso continuo de los bosques o ecosistemas forestales, así como el mantenimiento de su salud, productividad, diversidad e integridad ecológica a perpetuidad, para satisfacer las necesidades sociales, económicas, físicas y espirituales del hombre, tanto de las generaciones actuales como de las futuras. La Organización de las Naciones Unidas para la Alimentación y la Agricultura [FAO] (2003) indica sobre el tema, que el manejo forestal sustentable tiene como objetivo asegurar que los bienes y servicios derivados del mismo satisfagan las necesidades presentes a la vez que aseguren su disponibilidad continua y aporten al desarrollo a largo plazo. Herrera-Ulloa, Lluch-Cota, Ramírez-Aguirre, Hernández-Vázquez y Ortega-Rubio (2003) por su parte, sostienen que medir el desarrollo sustentable es una de las metas para un mejor desempeño de cualquier productor que hace uso de los recursos naturales y los indicadores son una forma para evaluar las decisiones políticas prácticas con las definiciones operacionales.

Las evaluaciones de sustentabilidad se han convertido en un área de intensa investigación a escala internacional enfocada a la definición de indicadores de sustentabilidad para la evaluación de sistemas desde los puntos de vista ambiental, económico y social (Masera, Astier y LópezRidaura, 1999). Sin embargo, según Sarandón (2002), uno de los problemas que surge cuando se intenta evaluar o medir la sustentabilidad, es la confusión respecto a qué es exactamente lo que se quiere evaluar.

Los indicadores pueden definirse como herramientas con las cuales se puede medir y evaluar el avance hacía el manejo forestal sustentable (Cano, Martínez, Martínez y Quiñones, 2007). Los indicadores son también una herramienta valiosa utilizada en la planeación, evaluación, manejo, inventario, monitoreo y comunicación. Ellos proporcionan un formato que soporta científicamente el manejo forestal y ayudan a la formulación de políticas efectivas (United States Department of Agriculture [USDA], 2002). Los indicadores de sustentabilidad se han convertido en una importante herramienta de toma de decisiones para gobiernos nacionales y locales, comunidades y actores sociales involucrados (Hammond, Adriaanse, Rodenburg, Bryant y Woodward, 1995).

Las evaluaciones de sustentabilidad mediante indicadores son resultado de abordar la realidad desde la perspectiva del desarrollo sustentable, haciendo un análisis de diferentes aspectos de una misma realidad, que posteriormente deben ser integrados para armar una explicación coherente y útil (Toledo, 1998). Un indicador de sustentabilidad, según sugiere Smith (2002), debe ser una medición simple, compuesta y numérica, que funcione 
como una clave o "apuntador" a la sustentabilidad de un sistema mayor. Esto provee bases para evaluar tendencias ambientales, establecer metas de políticas o monitorear progreso. Masera et al. (1999) afirman, asimismo, que un indicador describe un proceso específico o un proceso de control, por lo que debe ser construido de manera específica y siguiendo un proceso.

Hens y De Wit (2003) manifiestan que la experiencia ha mostrado que es científicamente factible establecer indicadores para medir el grado de sustentabilidad; generalmente, este proceso se inicia con el establecimiento de una gran lista de indicadores para describir el fenómeno tan complejo que es la sustentabilidad y, posteriormente, mediante procedimientos de participación u otros esta lista se reduce a una lista de indicadores estándar que pueden ser aplicados a diferentes países y en ocasiones a diferentes niveles, tales como: criminalidad, empleo, educación y esperanza de vida, de tal manera que se cumpla el propósito de proveer indicadores o el método para desarrollarlos en otros países. En la actualidad se han presentado múltiples estudios que tratan de evaluar el desempeño de la sustentabilidad mediante el uso de indicadores (índices) que ayudan a relacionar el término sustentabilidad con los cambios sociales y ambientales (Morse, McNamara y Acholo, 2004). En el contexto forestal, Prabhu et al. (1996) sostienen que diversos sistemas de indicadores de sustentabilidad a escala local (unidades de manejo forestal), han sido desarrollados por organizaciones como el Centro Internacional para la Investigación Forestal (Cifor), la Alianza de Bosques Tropicales, la Asociación de Suelos, la Organización Africana de la Madera, y la Propuesta de Tarapoto. En tal sentido, y dada la inexistencia de indicadores que permitan evaluar la sustentabilidad ambiental de las concesiones forestales en el departamento Huánuco, Perú, se ha visto por conveniente construir y validar indicadores que permitan evaluar la sustentabilidad ambiental de las concesiones forestales en estudio, para cuyo efecto se realizó una adaptación a la metodología y el marco conceptual propuesto por Sarandón (2002) y Sarandón y Flores (2009).
Según la Ley Forestal y de Fauna Silvestre $\left(\right.$ Ley N ${ }^{\circ}$ 29763), Artículo 51: "La concesión forestal es un bien incorporal registrable. Puede ser objeto de hipoteca, así como de disposición a través de la figura de cesión de posición contractual u otros actos acordes a la naturaleza del título. La concesión forestal, su disposición y la constitución de derechos reales sobre ella se inscriben en el registro público respectivo". La referida Ley establece, asimismo, que la explotación forestal en el Perú se rige bajo el sistema de concesiones.

Sarandón (2002) afirma que es importante entender qué es exactamente un indicador, al que define como una variable seleccionada y cuantificada que permite ver una tendencia que de otra forma no es fácilmente detectable. No obstante, refiere que lamentablemente no se dispone de este tipo de indicadores para la evaluación de la sustentabilidad de los agroecosistemas, por lo que se hace necesario desarrollarlos de la mejor manera posible. En general, según Sarandón (2002), existe acuerdo sobre algunos principios que deben cumplirse para un manejo sustentable de tierras. Uno de estos manejos es la agricultura que, por lo tanto, deberá ser ecológicamente adecuada (que conserve la base de recursos naturales y preserve la integridad del ambiente a escalas local, regional y global). En este contexto, los indicadores deberían evaluar o abarcar los siguientes aspectos ecológicos que afectan a: la capacidad productiva del agroecosistema (aspectos del manejo que ocasionen un cambio en la capacidad o potencial productivo del propio sistema, lo cual implica que los recursos renovables deben ser utilizados a un ritmo menor o igual al de su reposición y los recursos no renovables a un ritmo similar al que permita el desarrollo de una tecnología de sustitución del recurso), y el impacto ambiental externo al predio (aquellos aspectos que, aunque no atentan contra la productividad del sistema, causan un daño al ambiente o a la salud de animales y/o de la población en el corto o largo plazo). Pokorny, Sabogal y de Camino (2001) refieren, asimismo, que muchos de los usuarios no especialistas tienen dificultades en entender y usar adecuadamente la estructuración jerárquica de los criterios e indicadores. 
De lo anteriormente tratado, ante la ausencia de investigación acerca de indicadores que permitan determinar la sustentabilidad ambiental de las concesiones forestales en el departamento Huánuco, Perú y por la importancia de conocer si realmente el aprovechamiento forestal es sustentable acorde a la legislación vigente, la presente investigación constituye un aporte real que podría ser de utilidad para la toma de decisiones en las políticas forestales del departamento Huánuco y que, a su vez, pueda convertirse en el punto de partida para estudios en otras regiones del país.

\section{OBJETIVOS}

El objetivo del presente estudio fue determinar la sustentabilidad ambiental de las concesiones forestales ubicadas en el departamento Huánuco, Perú, mediante el análisis multicriterio basado en el uso de indicadores de sustentabilidad ambiental. Para su consecución se tuvo previamente, que construir y luego validar los indicadores en referencia.

\section{MATERIALES Y MÉTODOS}

\section{Área de estudio}

La investigación se desarrolló en cuatro provincias del departamento Huánuco, donde se localizan las concesiones forestales activas (Tabla 1, Fig. 1). Cabe destacar que, en el Perú, de acuerdo con la actual Ley Forestal y de Fauna
Silvestre (Ley N² 29763, 2011) y mediante las concesiones forestales, el Estado otorga, en áreas de dominio público, derecho para el aprovechamiento sostenible de los recursos forestales y de fauna silvestre, preservando su riqueza, evitando daños al ecosistema y propiciando la regeneración natural de los árboles. La referida ley otorga concesiones forestales con fines maderables por un plazo de hasta cuarenta años renovables en bosques de producción permanente (BPP), establecidos en bosques primarios o secundarios y con dos categorías: categoría I, para extracción de madera bajo sistemas intensivos o mecanizados y categoría II, para extracción de madera solo bajo sistemas de baja intensidad. Las concesiones se obtienen a través de concurso público en unidades de aprovechamiento de cinco mil hasta diez mil hectáreas, y en unidades de aprovechamiento de más de diez mil hasta cuarenta mil hectáreas.

Según estudios realizados por Gobierno Regional de Huánuco [Gorehco] (2016), la provincia de Leoncio Prado se encuentra a una altitud que oscila entre los $500 \mathrm{~m}$ y 1500 m s. n. m. La precipitación pluvial adquiere valores entre $2000 \mathrm{~mm} /$ año y $3500 \mathrm{~mm} /$ año. La temperatura mínima promedio anual es de $18.7^{\circ} \mathrm{C}$ y la máxima promedio anual es de $29.5^{\circ} \mathrm{C}$. Asimismo, Gorehco (2016) tomando como base la clasificación climática propuesta por Thornthwaite (1948), encontró los siguientes tipos de clima: $\mathrm{B}_{4} \mathrm{rA}$ 'a', $\mathrm{B}_{3} \mathrm{rB}_{4}{ }_{4 a}, \mathrm{~B}_{2} \mathrm{rB}_{4}{ }_{4 a} \mathrm{y}^{\prime}$ y $\mathrm{ArA} \mathrm{A}^{\prime}$ '.

TABLA 1. Ámbito de acción de la investigación.

\begin{tabular}{|c|c|c|c|c|}
\hline Departamento & Provincia & Distrito & Código de la concesión forestal & Área (ha) \\
\hline \multirow{9}{*}{ Huánuco } & \multirow{3}{*}{ Leoncio Prado } & José Crespo y Castillo & $\mathrm{CF} 1$ & 5744 \\
\hline & & José Crespo y Castillo & \multirow{2}{*}{ CF2 } & \multirow{2}{*}{5068} \\
\hline & & Pucayacu & & \\
\hline & \multirow{3}{*}{ Leoncio Prado } & José Crespo y Castillo & \multirow{4}{*}{ CF3 } & \multirow{4}{*}{27978} \\
\hline & & Pueblo Nuevo & & \\
\hline & & Rupa Rupa & & \\
\hline & Huamalíes & Monzón & & \\
\hline & Marañón & La Morada & CF4 & 8272 \\
\hline & Puerto Inca & Codo del Pozuzo & CF5 & 7700 \\
\hline & & Área total (ha) & & 54762 \\
\hline
\end{tabular}




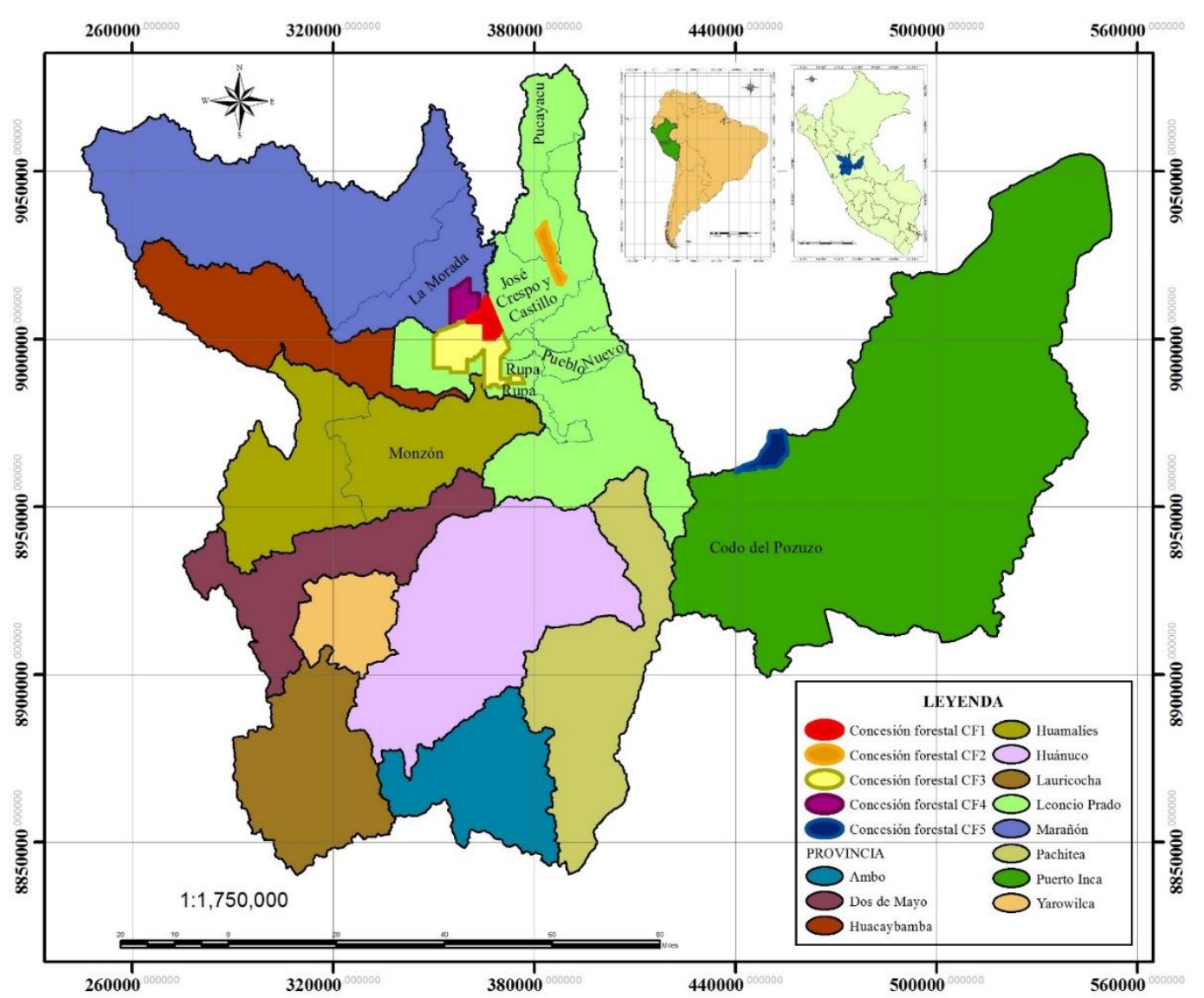

FIgURA 1. Ámbito de acción de la investigación.

En la provincia de Marañón, Gorehco (2016a) refiere que su altitud varía entre $850 \mathrm{~m}$ y $5650 \mathrm{~m}$ s. n. m. La precipitación pluvial oscila entre $1000 \mathrm{~mm}$ /año y 2500 $\mathrm{mm} /$ año. El promedio de la temperatura mínima anual es de $6{ }^{\circ} \mathrm{C}$ y el promedio anual de la temperatura máxima es de $28{ }^{\circ} \mathrm{C}$. Así también, Gorehco (2016a) tomando como referencia la clasificación climática sugerida por Thornthwaite (1948), indica la presencia de los siguientes tipos de clima: $\mathrm{B}_{4} \mathrm{rA}^{\prime} \mathrm{a}^{\prime}, \mathrm{B}_{3} \mathrm{rB}_{4}{ }_{4} \mathrm{a}^{\prime}, \mathrm{B}_{2} \mathrm{rB}^{\prime}{ }_{4 a} \mathrm{a}^{\prime}, \mathrm{C}_{2} \mathrm{rB}^{\prime}{ }_{1} \mathrm{a}^{\prime}$ y $\mathrm{C}_{1} \mathrm{~dB}_{2}{ }_{2}{ }^{\prime}$. La provincia de Puerto Inca presenta una altitud comprendida entre los $250 \mathrm{~m}$ y $770 \mathrm{~m}$ s.n.m. La precipitación pluvial varía entre $2000 \mathrm{~mm} /$ año y 3500 $\mathrm{mm}$ /año. La temperatura mínima promedio anual es de 25 ${ }^{\circ} \mathrm{C}$ y la máxima promedio anual es de $30.9^{\circ} \mathrm{C}$ (Gorehco, 2016b). Por su parte, Gorehco (2016b) basado en la clasificación climática establecida por Thornthwaite (1948), encontró los siguientes tipos de clima: B 4 rA'a' y ArA'a'.
Gorehco (2016) sostiene así también, que las provincias de Leoncio Prado, Marañón y Puerto Inca se caracterizan por su variada geografía y su ingente riqueza en recursos naturales, destacándose su capacidad hídrica y forestal, factores que le permiten contar con un gran potencial de crecimiento económico. En efecto, los diversos pisos ecológicos y su clima resultan aptos para el desarrollo de múltiples cultivos, desde frutales tropicales en la zona oriental, hasta los típicamente andinos en la sierra. Por otro lado, la región cuenta con extensos bosques y áreas aptas para reforestación lo que le confiere una clara aptitud para el desarrollo de la industria forestal.

La provincia de Huamalíes según Meza y Díaz (2010), se localiza a una altitud de entre $680 \mathrm{~m}$ y $4950 \mathrm{~m}$ s.n.m. La precipitación pluvial presenta valores de entre $500 \mathrm{~mm} /$ año y $2000 \mathrm{~mm} /$ año. La temperatura mínima anual promedio es de $6{ }^{\circ} \mathrm{C}$ y la máxima anual promedio es de $22{ }^{\circ} \mathrm{C}$. 
Asimismo, indican que tomando como referencia la clasificación climática de Köppen, en la provincia de Huamalíes se identifican cuatro tipos: clima subtropical muy húmedo (Aw), clima templado subhúmedo (BSw), clima frío o boreal (Dwb) y clima frígido (ETH).

\section{Tamaño de la población}

Las concesiones forestales activas y operativas al momento del desarrollo de la investigación, fueron cinco. En tal sentido, se realizó el estudio en la totalidad de ellas.

\section{Construcción de indicadores}

Luego de haber estructurado, estandarizado y ponderado de manera preliminar los indicadores de sustentabilidad, se desarrolló en la Universidad Nacional Agraria de la SelvaTingo María, un Taller para la validación de indicadores de sustentabilidad y encuestas para las concesiones forestales del departamento buánuco, con participación de titulares de cuatro concesiones forestales; funcionarios de la Administración Técnica Forestal y Fauna Silvestre (ATFFS); y egresados, estudiantes y docentes de la Escuela Profesional de Ingeniería Forestal (EPIF) de la Facultad de Recursos Naturales Renovables de la Universidad Nacional Agraria de la Selva. Para el efecto, se siguieron los pasos propuestos por Sarandón (2002) y Sarandón y Flores (2009), a los que fue necesario realizar diversas adaptaciones por tratarse de una investigación en el contexto forestal. Se eligieron indicadores fáciles de obtener e interpretar y que, a su vez brindaron, la información que permitió detectar tendencias en la concesión forestal. Estos estuvieron compuestos a su vez, por subindicadores.

\section{Estandarización de los indicadores}

La estandarización consistió en asignar un valor numérico a cada uno de los parámetros relacionados directamente con cada dimensión de la sustentabilidad. A fin de permitir la comparación de las fincas y facilitar el análisis de las múltiples dimensiones de la sustentabilidad, los datos fueron estandarizados mediante su transformación a una escala para cada indicador (de 0 a 4 ), siendo 4 el mayor valor de sustentabilidad y 0 el más bajo valor. Todos los valores, independientemente de su unidad original, se transformaron o adecuaron a esta escala. Esto posibilitó la integración de varios indicadores de distinta naturaleza, en otros más sintéticos o robustos. Los indicadores ambientales priorizados se detallan en la tabla 2.

Posteriormente, los indicadores fueron ponderados multiplicando el valor de la escala por un coeficiente de acuerdo con la importancia relativa de cada variable respecto a la sustentabilidad. Este coeficiente multiplica tanto el valor de las variables que forman el indicador, como el de los propios indicadores, para construir otros de mayor nivel o índices. La ponderación es un paso inevitable y fue realizada por consenso, por medio de la consulta con expertos en el tema, según lo propuesto por Gayoso e Iroumé (1991) y teniendo en cuenta la opinión de los propios agricultores o titulares de concesión forestal para la presente investigación, de acuerdo con lo sugerido por Lefroy, Bechstedt y Rais (2000). En la presente investigación, el indicador C (conservación y mantenimiento del recurso suelo) recibió una ponderación de 2, debido a su importancia como base de toda actividad productiva.

El grado de cumplimiento de la dimensión ambiental (IA) está definido por el indicador cuyo valor máximo es de 4 y se considera sustentable cuando es igual o mayor a 2 . Su cálculo fue realizado mediante la fórmula:

Indicador Ambiental (IA) =

$\frac{\mathrm{A}+\mathrm{B}+2\left(\frac{\mathrm{C} 1+\mathrm{C} 2+\mathrm{C} 3+\mathrm{C} 4+\mathrm{C} 5}{5}\right)+\mathrm{D}+\mathrm{E}}{6}$

\section{Diseño de encuestas y toma de datos}

Las encuestas fueron elaboradas empleando dos diseños distintos; para los titulares de concesiones forestales tuvo 63 preguntas y, para los trabajadores, 10 preguntas. En ambos casos, se buscó que se adaptaran a la obtención de información de unidades de aprovechamiento diferentes a fincas agropecuarias (concesiones forestales). Posteriormente, se aplicó la encuesta a los cinco titulares de concesiones forestales y a 27 trabajadores; es decir, a 32 personas en total. 
TABLA 2. Indicadores ambientales priorizados.

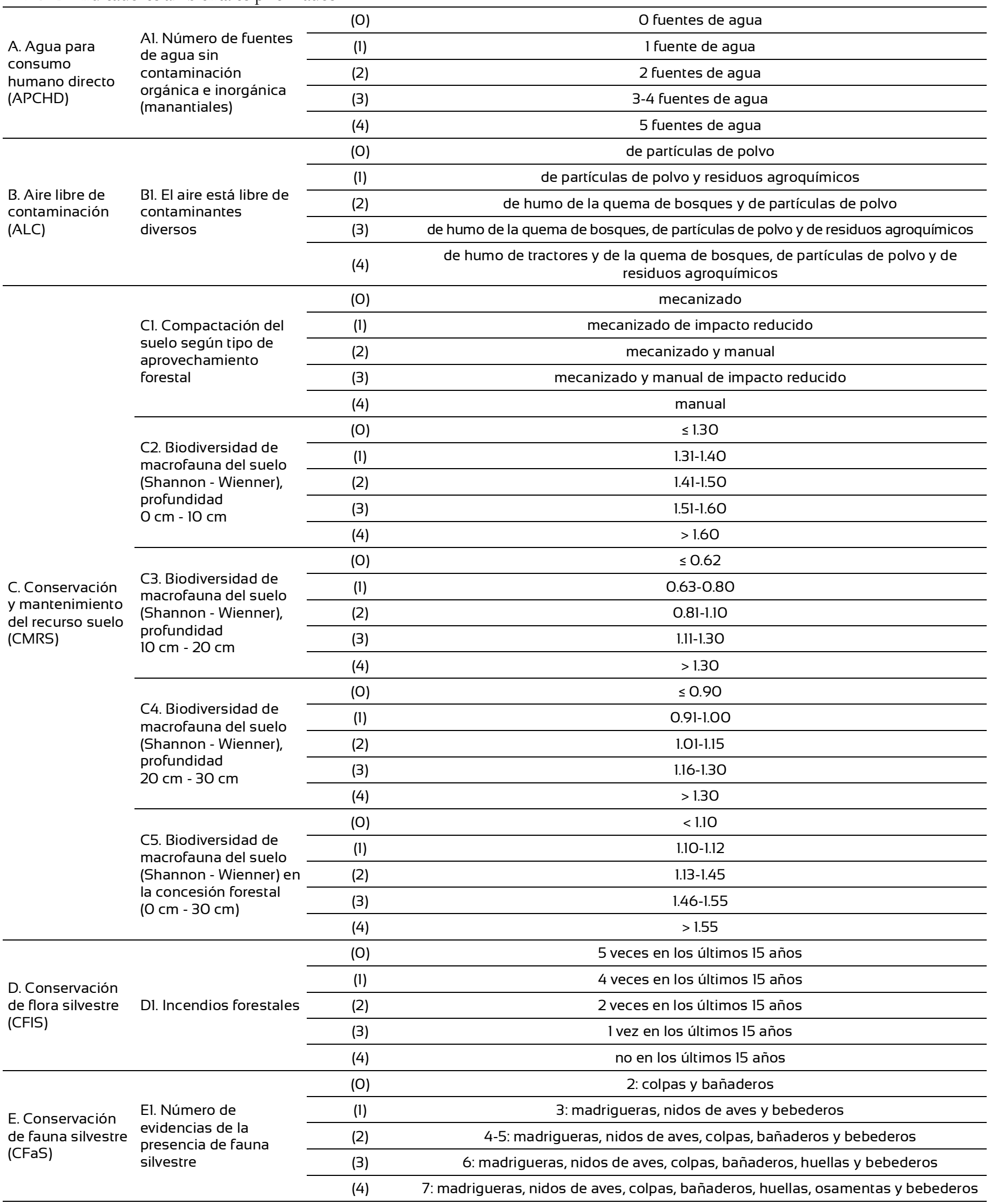


No obstante, con la finalidad de construir un indicador ambiental que muestrara el estado de conservación y mantenimiento del suelo sometido a aprovechamiento forestal, se procedió a la colecta de organismos que constituyen la macrofauna del suelo, hasta una profundidad de $30 \mathrm{~cm}$, empleando el método de muestreo recomendado por el Programa Tropical Soil Biology and Fertility (TSBF) (Anderson e Ingram, 1993). Se tomaron cinco muestras en un área aprovechada de 1 ha en cada concesión forestal, mismas que fueron distribuidas cada 10 metros en línea recta, al centro de la parcela de una hectárea y en sentido de la pendiente. En cada una de ellas se procedió a extraer mediante un marco metálico de $25 \mathrm{~cm} \times 25 \mathrm{~cm} \times 10 \mathrm{~cm}$ de profundidad, tres submuestras (en total se obtuvieron 75 submuestras). La macrofauna fue expresada en términos de diversidad, de acuerdo con el índice de Shannon-Wiener. Los valores obtenidos fueron posteriormente estandarizados y ponderados simultáneamente con el resto de los indicadores ambientales.

\section{Análisis estadístico}

Se aplicó la prueba chi cuadrada, con la finalidad de conocer si el índice de sustentabilidad ambiental en $60 \%$ de las concesiones forestales en estudio supera el valor de 2.0, considerándose a la totalidad de ellas como una muestra. En consecuencia, se plantearon las siguientes hipótesis:

$\mathrm{H}_{0}$ : La proporción de concesiones forestales cuyo índice de sustentabilidad ambiental supera a 2.0, es igual a $60 \%$ de las mismas.

$\mathrm{H}_{1}$ : La proporción de concesiones forestales cuyo índice de sustentabilidad ambiental supera a 2.0, no es igual a $60 \%$ de las mismas.

La toma de decisión se realizó con el p-valor obtenido. Posteriormente, se desarrollaron los supuestos de normalidad entre los indicadores de sustentabilidad ambiental, para luego proceder a aplicar la correlación de Pearson (r) con 95\% de nivel de confiabilidad, con el propósito de determinar la existencia de correlación entre ellos; la escala corresponde a la propuesta por Hernández (2014).

\section{Resultados}

\section{Sustentabilidad ambiental de las concesiones forestales en el departamento Huánuco}

Concluida la evaluación de los indicadores de sustentabilidad ambiental para las concesiones forestales, se obtuvieron valores del índice de sustentabilidad ambiental mayores a 2.0 en las tres concesiones forestales cuyo aprovechamiento forestal se realiza de manera manual (Tabla 3; Fig. 2a; Fig. 2b; Fig. 2c; Fig. 3); es decir, 60\% de las concesiones en estudio son ambientalmente sustentables. No obstante, se observa que las dos concesiones forestales que realizan aprovechamiento forestal mecanizado $(40 \%$ del total), resultan no sustentables ambientalmente (Tabla 3). Las figuras $2 \mathrm{~d}$, 2e y 3 evidencian que la mayor cantidad de indicadores adquieren valores por debajo del umbral de sustentabilidad (menores a 2.0).

La prueba chi cuadrada evidenció que, de acuerdo con el p-valor obtenido, se acepta la hipótesis nula $\left(\mathrm{H}_{0}\right)\left(\mathrm{X}^{2}=\right.$ 0.056 , p-valor $=0.814$, , con lo cual se confirma que en $60 \%$ de concesiones forestales el índice de sustentabilidad ambiental supera al valor de 2.0; es decir, son sustentables ambientalmente las concesiones que realizan aprovechamiento forestal manual (Tabla 4).

La correlación de Pearson muestra una relación positiva estadísticamente significativa $(\mathrm{r}=0.970, \mathrm{p}$-valor $<$ $0.05)$ entre los indicadores "conservación y mantenimiento del recurso suelo" y "conservación de flora silvestre"; dicha correlación se clasifica como fuerte, en tanto los indicadores restantes son independientes (Tabla 5). 
TABLA 3. Sustentabilidad ambiental de las concesiones forestales del departamento Huánuco, Perú

\begin{tabular}{|c|c|c|c|c|c|c|}
\hline \multirow{2}{*}{ Indicadores } & \multirow{2}{*}{ Subindicadores } & \multicolumn{5}{|c|}{ Concesión forestal } \\
\hline & & $\mathrm{CF} 1$ & CF2 & CF3 & CF4 & CF5 \\
\hline \multirow[t]{2}{*}{$\begin{array}{c}\text { Agua para consumo } \\
\text { humano directo-APCHD } \\
\text { (A) }\end{array}$} & $\begin{array}{c}\text { FASCOI. Número de fuentes de agua } \\
\text { sin contaminación orgánica e } \\
\text { inorgánica (manantiales) } \\
\text { (Al) }\end{array}$ & 1 & 3 & 4 & 0 & 2 \\
\hline & APCHD & 1 & 3 & 4 & 0 & 2 \\
\hline \multirow[t]{2}{*}{$\begin{array}{c}\text { Aire libre de } \\
\text { contaminación-ALC } \\
\text { (B) }\end{array}$} & $\begin{array}{c}\text { ALCD. El aire está libre de } \\
\text { contaminantes diversos } \\
\text { (B1) }\end{array}$ & 4 & 3 & 1 & 2 & 0 \\
\hline & ALC & 4 & 3 & 1 & 2 & 0 \\
\hline \multirow{6}{*}{$\begin{array}{c}\text { Conservación y } \\
\text { mantenimiento del recurso } \\
\text { suelo-CMRS } \\
\text { (C) }\end{array}$} & $\begin{array}{l}\text { CSSTEF. Compactación del suelo } \\
\text { según tipo de aprovechamiento } \\
\text { forestal } \\
\text { (C1) }\end{array}$ & 4 & 2 & 3 & 1 & 0 \\
\hline & $\begin{array}{l}\text { BMS 10. Biodiversidad de macrofauna } \\
\text { del suelo (Shannon - Wienner), } \\
\text { profundidad } 0 \mathrm{~cm}-10 \mathrm{~cm} \\
\text { (C2) }\end{array}$ & 2 & 3 & 4 & 1 & 0 \\
\hline & $\begin{array}{c}\text { BMS 20. Biodiversidad de } \\
\text { macrofauna del suelo (Shannon - } \\
\text { Wienner), profundidad } 10 \mathrm{~cm}-20 \mathrm{~cm} \\
\text { (C3) }\end{array}$ & 3 & 4 & 2 & 1 & 0 \\
\hline & $\begin{array}{c}\text { BMS 30. Biodiversidad de } \\
\text { macrofauna del suelo (Shannon - } \\
\text { Wienner), profundidad } 20 \mathrm{~cm}-30 \mathrm{~cm} \\
\text { (C4) }\end{array}$ & 0 & 3 & 4 & 2 & 1 \\
\hline & $\begin{array}{l}\text { BMS 0-30. Biodiversidad de } \\
\text { macrofauna del suelo (Shannon - } \\
\text { Wienner) en la concesión forestal (O } \\
\mathrm{cm}-30 \mathrm{~cm} \text { ) } \\
\text { (C5) }\end{array}$ & 2 & 4 & 3 & 1 & 0 \\
\hline & CMRS & 2.2 & 3.2 & 3.2 & 1.2 & 0.2 \\
\hline \multirow{2}{*}{$\begin{array}{l}\text { Conservación de flora } \\
\text { silvestre-CFIS } \\
\text { (D) }\end{array}$} & $\begin{array}{l}\text { IF. Incendios forestales } \\
\text { (D1) }\end{array}$ & 2 & 4 & 3 & 1 & $\mathrm{O}$ \\
\hline & CFIS & 2 & 4 & 3 & 1 & 0 \\
\hline \multirow{2}{*}{$\begin{array}{l}\text { Conservación de fauna } \\
\text { silvestre-CFaS } \\
\text { (E) }\end{array}$} & $\begin{array}{l}\text { EPFS. Número de evidencias de la } \\
\text { presencia de fauna silvestre } \\
\text { (E1) }\end{array}$ & 2 & 3 & 0 & 1 & 4 \\
\hline & CFaS & 2 & 3 & 0 & 1 & 4 \\
\hline $\begin{array}{c}\text { Índice de Sustentabilidad } \\
\text { Ambiental (ISA) }\end{array}$ & & 2.23 & 3.23 & 2.4 & 1.07 & 1.07 \\
\hline
\end{tabular}




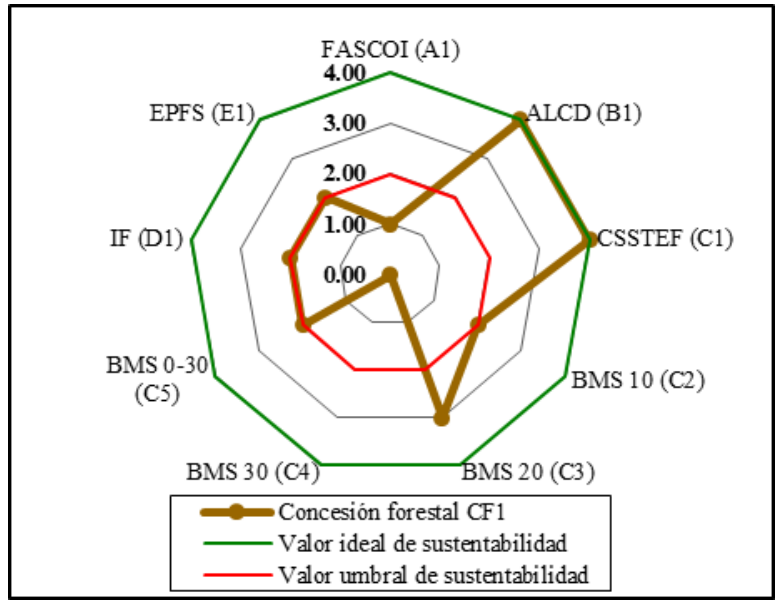

a)

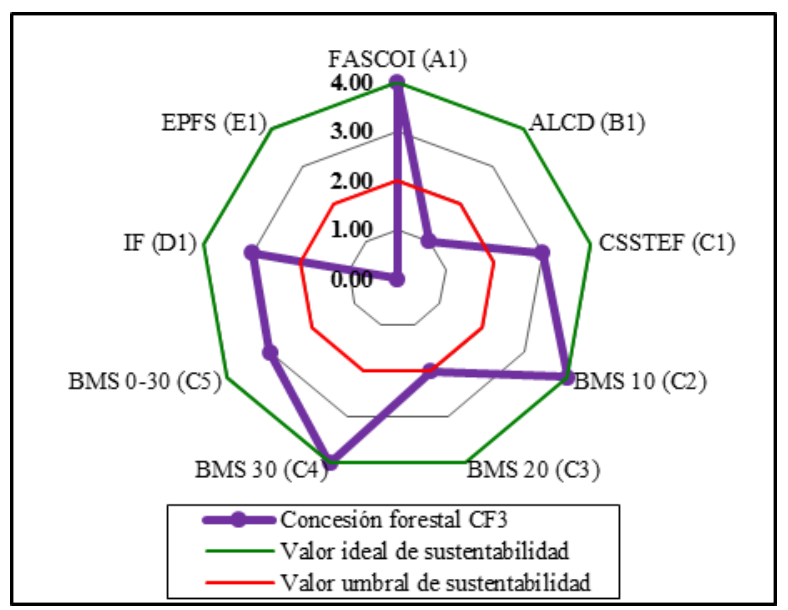

c)

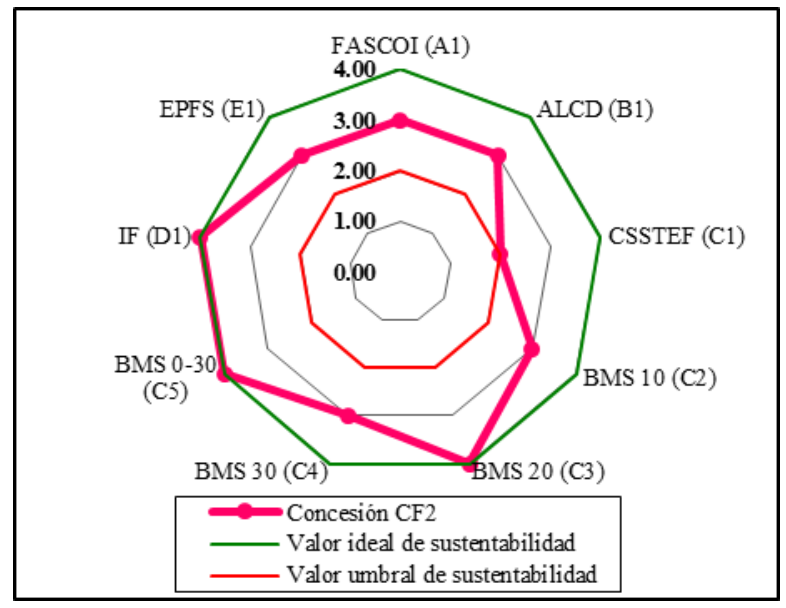

b)

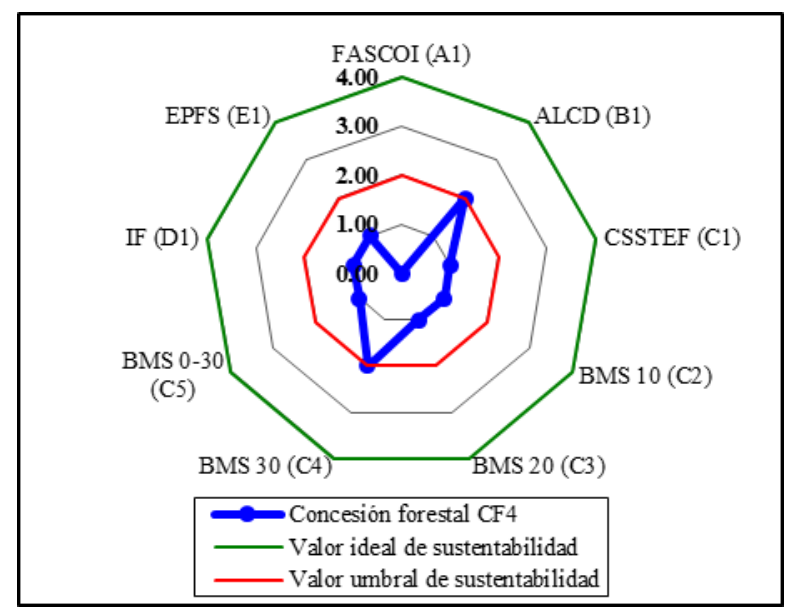

d)

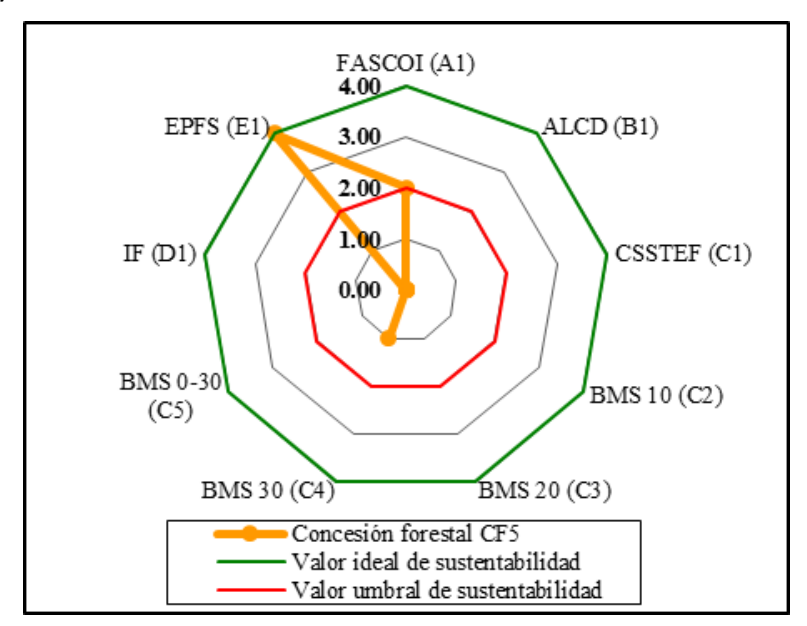

e)

FIGURA 2. Subindicadores de sustentabilidad ambiental para las cinco concesiones forestales estudiadas.

FASCOI: Número de fuentes de agua sin contaminación orgánica e inorgánica (manantiales); ALCD: El aire está libre de contaminantes diversos; CSSTEF: Compactación del suelo según tipo de aprovechamiento forestal; BMS 1O: Biodiversidad de macrofauna del suelo (Shannon - Wienner), profundidad O cm - $10 \mathrm{~cm}$; BMS 20 : Biodiversidad de macrofauna del suelo (Shannon - Wienner), profundidad $10 \mathrm{~cm}-20 \mathrm{~cm}$; BMS 30: Biodiversidad de macrofauna del suelo (Shannon - Wienner), profundidad $20 \mathrm{~cm}$ - 30 cm; BMS O-30: Biodiversidad de macrofauna del suelo (Shannon - Wienner) en la concesión forestal; IF: Incendios forestales; EPFS: Número de evidencias de la presencia de fauna silvestre. 


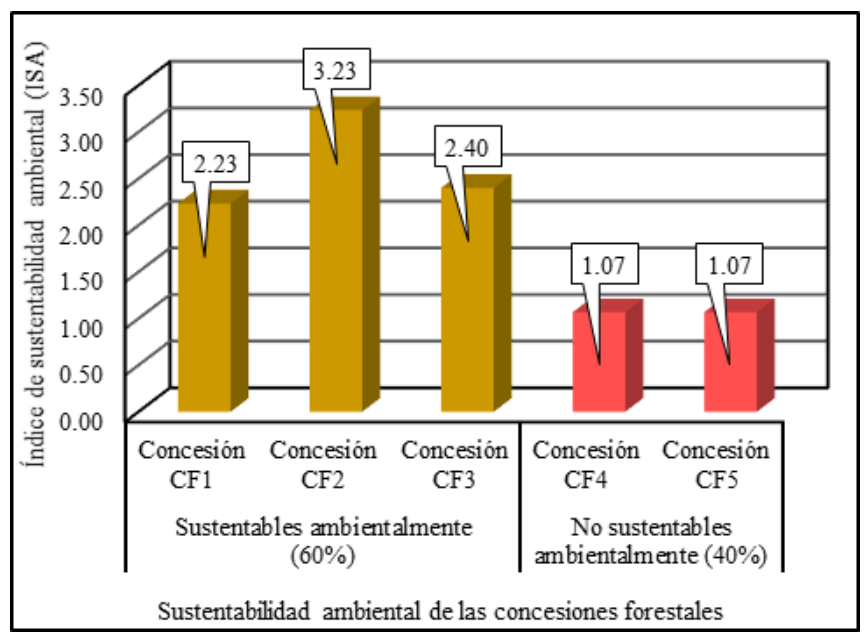

FIGURA 3. Sustentabilidad de concesiones forestales.

TABLA 4. Prueba chi cuadrada para las proporciones del índice de sustentabilidad ambiental.

\begin{tabular}{cc}
\hline Parámetros & Sustentable ambientalmente \\
\hline $\begin{array}{c}\text { Chi cuadrada } \\
\left(\mathrm{X}^{2}\right)\end{array}$ & 0.056 \\
\hline $\mathrm{GL}$ & 1.00 \\
\hline $\mathrm{P}$-valor & $0.814^{\mathrm{ns}}$ \\
\hline
\end{tabular}

ns: No existe significancia estadística.

\section{DISCUSIÓN}

Las concesiones forestales CF1, CF2 y CF3, cuyo aprovechamiento forestal se realiza de manera manual, son sustentables ambientalmente. La mayor cantidad de indicadores ambientales adquieren valores iguales $\mathrm{y}$ mayores a 2.0; solo para el caso de la concesión forestal CF1, el indicador "agua para consumo humano directo" (APCHD) obtuvo un valor de 1 y para la concesión forestal $\mathrm{CF} 3$ se obtuvo un valor de 1 para el indicador "aire libre de contaminación" (ALC) y 0 para el indicador "conservación de fauna silvestre" (CFaS). Ello constituye una clara evidencia de que el aprovechamiento forestal manual es menos invasivo y mantiene armonía con el entorno natural; sobre el tema, Contreras, Cordero y Fredericksen (2001) refieren que toda actividad de aprovechamiento forestal ocasionará, inevitablemente, algún nivel de daño ya sea a la masa remanente, al suelo y/o a las fuentes de agua.

Cruz (2003) manifiesta también que las actividades de aprovechamiento forestal pueden realizarse de manera artesanal o manual y que por lo general no ocasionan daños significativos en comparación con el uso de maquinaria. Por su parte, Akay, Yilmaz y Tongue (2006) refieren que, una vez que los aprovechamientos forestales se tecnificaron, se reconocieron los impactos en los árboles remanentes y en el suelo; y según Solgi y Najafi (2007), cada sistema incide de distinta manera: la mala aplicación y supervisión de las operaciones de apertura de caminos, derribo y arrastre ocasionan serios daños en los bosques naturales, debido a la existencia de especies y árboles de diversas edades.

Lo referido anteriormente fundamenta los resultados obtenidos por las tres concesiones forestales que practican aprovechamiento forestal manual, dado que ambientalmente resultan sustentables debido a que pese a causar algún tipo de daño inevitable a los diversos recursos naturales, este impacto es menor comparado con el ocasionado por el aprovechamiento forestal mecanizado.

Resulta necesario, entonces, conocer la base de los nuevos paradigmas del manejo forestal sustentable. Estas, según Hawksworth y Bull (2006) y Grossberg (2009), están dadas por las evidencias de los beneficios ecológicos, económicos y sociales de los nuevos modelos de manejo, que dejan claro que no se trata de optar entre desarrollo y conservación, sino que existen respuestas posibles desde las personas que habitan los bosques para cumplir los objetivos del desarrollo sostenible en las regiones forestales.

Se observa por otra parte, que las concesiones forestales CF4 y CF5 resultaron no sustentables ambientalmente, obteniendo un índice de sustentabilidad ambiental menor a 2.0. Para el caso de la primera concesión forestal, los indicadores "agua para consumo humano directo" (APCHD), "conservación de flora silvestre" (CFIS) y "conservación de fauna silvestre" (CFaS) alcanzaron valores entre 0 y 1.0 , pese a realizar aprovechamiento forestal mecanizado de impacto reducido. Por otra parte, la concesión forestal CF5 presenta valores entre 0 y 0.2 para los indicadores "aire libre de contaminación" (ALC), "conservación de flora silvestre" (CFIS) y "conservación y mantenimiento del recurso suelo" (CMRS), lo cual se ve acentuado por el aprovechamiento forestal mecanizado que se practica. 
TABLA 5. Correlación entre los indicadores de la sostenibilidad ambiental en las concesiones forestales.

\begin{tabular}{|c|c|c|c|c|c|c|}
\hline & Variables & APCHD & ALC & CMRS & CFIS & CFaS \\
\hline \multirow{3}{*}{ APCHD } & Correlación de Pearson & 1 & -0.300 & 0.606 & 0.600 & -0.100 \\
\hline & p-valor & & $0.624^{\mathrm{ns}}$ & $0.278^{\text {ns }}$ & $0.285^{\text {ns }}$ & $0.873^{n s}$ \\
\hline & $\mathrm{N}$ & 5 & 5 & 5 & 5 & 5 \\
\hline \multirow{3}{*}{ ALC } & Correlación de Pearson & -0.300 & 1 & 0.485 & 0.500 & -0.100 \\
\hline & $\mathrm{p}$-valor & $0.624^{\mathrm{ns}}$ & & $0.408^{\text {ns }}$ & $0.391^{\text {ns }}$ & $0.873^{\text {ns }}$ \\
\hline & $\mathrm{N}$ & 5 & 5 & 5 & 5 & 5 \\
\hline \multirow{3}{*}{ CMRS } & Correlación de Pearson & 0.606 & 0.485 & 1 & 0.970 & -0.485 \\
\hline & p-valor & $0.278^{\text {ns }}$ & $0.408^{\text {ns }}$ & & $0.006^{* *}$ & $0.408^{\text {ns }}$ \\
\hline & $\mathrm{N}$ & 5 & 5 & 5 & 5 & 5 \\
\hline \multirow{3}{*}{ CFIS } & Correlación de Pearson & 0.600 & 0.500 & 0.970 & 1 & -0.300 \\
\hline & $\mathrm{p}$-valor & $0.285^{n s}$ & $0.391^{\text {ns }}$ & $0.006^{* *}$ & & $0.624^{\mathrm{ns}}$ \\
\hline & $\mathrm{N}$ & 5 & 5 & 5 & 5 & 5 \\
\hline \multirow{3}{*}{ CFaS } & Correlación de Pearson & -0.100 & -0.100 & -0.485 & -0.300 & 1 \\
\hline & p-valor & $0.873^{\text {ns }}$ & $0.873^{\text {ns }}$ & $0.408^{\text {ns }}$ & $0.624^{\mathrm{ns}}$ & \\
\hline & $\mathrm{N}$ & 5 & 5 & 5 & 5 & 5 \\
\hline
\end{tabular}

APCHD: Agua para consumo humano directo, ALC: Aire libre de contaminación, CMRS: Conservación y mantenimiento del recurso suelo, CFIS: Conservación de flora silvestre, CFaS: Conservación de fauna silvestre.

**: La correlación es significativa en el nivel O.Ol (bilateral).

ns: No existe correlación estadística significativa.

La compactación del suelo, según Hildebrand y SchackKirchner (2002), aumenta con la presión ejercida por los tractores o animales, la frecuencia de su paso por un determinado punto y el arrastre de madera al ser transportado en suspensión. Al parecer, la humedad es el factor decisivo en la magnitud del fenómeno.

Orozco et al. (2006) mencionan que la infiltración de la precipitación y el consecuente flujo de agua en el subsuelo son muy importantes para la calidad de agua, porque por un lado evitan la escorrentía y, por otro, evitan el contacto con otros organismos ya que el suelo actúa como filtro para retener organismos posibles contaminantes o exceso de minerales en el agua. Lozada y Arends (1998) señalan al respecto, que todas las maquinarias necesitan cambios de lubricantes, reposición de combustibles y ocasionalmente lavados que se hacen con detergente y kerosene; en muchas oportunidades estas operaciones se hacen en montaña y generan residuos que contaminan las aguas.

Por otra parte, diversos investigadores sugieren que la extracción de madera de bosques naturales genera cambios sustanciales sobre su estructura y composición, y la consecuente pérdida de hábitats y recursos indispensables para la supervivencia de la fauna que en ellos habita, especialmente aquella que depende del dosel (Fredericksen, 2000; Padmawathe, Qureshi y Rawat, 2004). Cabe acotar que la cosecha de árboles se denomina corta comercial, si se retira la mayor parte del arbolado, y corta selectiva o por entresaca, si solo se talan los árboles más valiosos y se deja un número importante de los de menor valor. Este número dependerá de la edad y de la estructura del bosque, así como de consideraciones económicas. Muchos de los árboles que se dejan, caerán ante la fuerza del viento o el peso del hielo y la nieve una vez se les haya privado del soporte que presentaban los árboles talados y se hallen expuestos a los elementos (Chen, Franklin y Spies, 1995). En mérito a lo antes mencionado, precisa indicar que, en general, en el Perú el aprovechamiento forestal se caracteriza por la extracción selectiva de los árboles de especies más valiosas, lo cual, por cierto, es característica también de las concesiones forestales en estudio; por tal razón, el 
aprovechamiento forestal con fines maderables no constituye la principal causa de deforestación en el país. Ello concuerda con lo manifestado por la Consultora Internacional Independiente [Indufor] (2012), quien sostiene que las principales causas directas de la deforestación en el Perú son la agricultura y la ganadería, seguidas de la minería ilegal y los cultivos ilícitos e ilegales como la coca. Además, en menor proporción, existe deforestación por los proyectos de infraestructura como las hidroeléctricas, la explotación de hidrocarburos, las malas prácticas de extracción de madera y la tala ilegal. Zwane (2007) y Velarde et al. (2010), por su parte, refieren que en el Perú las causas subyacentes de la deforestación y la degradación de los bosques en la cuenca amazónica se deben principalmente a la migración de los campesinos de Los Andes a la Amazonía, favorecida con la construcción de carreteras de acceso desde la capital.

Se encontró, asimismo, según la correlación de Pearson, que los indicadores "conservación y mantenimiento del recurso suelo" (CMRS) y "conservación de flora silvestre" (CFIS) presentan una fuerte correlación positiva estadísticamente significativa (0.970). Al respecto, el coeficiente de correlación de Pearson, según Pearson (1920), mide el grado de correlación entre dos variables " $\mathrm{X}$ " y "Y", cuyos valores fluctúan desde -1 (correlación negativa perfecta de $100 \%$ ), hasta +1 (correlación positiva perfecta de 100\%). Cabe indicar que, en el caso de correlación negativa, un aumento en una variable se asocia con un decremento en otra variable, y en el caso a la correlación positiva ocurre lo contrario a lo mencionado anteriormente. Esta correlación paramétrica se aplica para casos en donde la distribución de los datos sigue una curva gausiana o normal. Es decir, se usa la correlación para estimar cuáles variables son potencialmente importantes, siendo el interés conocer el grado de la relación. De lo antes manifestado, se observa que los subindicadores "conservación y mantenimiento del recurso suelo" (CMRS) y "conservación de flora silvestre" (CFIS) son dos variables potencialmente importantes para la sustentabilidad ambiental de las concesiones forestales en el departamento Huánuco, donde a mayor conservación del recurso suelo, se logrará una mayor conservación de la flora silvestre, y viceversa, con un alto grado de relación entre ambas, clasificándola como correlación fuerte. Esta fuerte correlación positiva se manifiesta, por ejemplo, cuando la cubierta vegetal protege al suelo de la erosión al favorecer la infiltración del agua (Valentin, Poesen y Li, 2005) y a través del armado de las raíces, la intercepción de la precipitación y el incremento de la rugosidad superficial (Torri et al., 2013). La vegetación también afecta a las propiedades físicas, químicas e hidrológicas del suelo (Bierbaß, Wündsch y Michalzik, 2014), gracias al mayor espesor de mantillo (Navarro, de Araújo y Mongil, 2014) y hojarasca. Por consiguiente y según Zhang, Zhou, Cheng y Zhang (2013), en las zonas boscosas el suelo es de mejor calidad en comparación con el de las zonas degradadas sin reforestar. Por su parte, Bierbaß et al. (2014) refieren que la vegetación arbórea favorece que el agua se infiltre con mayor rapidez y a mayor profundidad. Respecto a la materia orgánica que se acumula bajo la cubierta vegetal, Neary, Ice y Jackson (2009) afirman que incrementa la fertilidad, la penetración de agua en el suelo y la retención de agua y sedimentos. Mackensen, Tillery-Stevens, Klinge y Fölster (2000) y Dezzeo, Chacón, Sanoja y Picón (2004) indican también, que la nutrición de las plantas depende estrictamente del ciclo de nutrientes en el ecosistema y la exuberancia de algunos bosques se sostiene por una fertilidad contenida en la biomasa.

Sin embargo, una perspectiva errónea del manejo forestal, centrada solo en el manejo de masas arboladas para la producción de madera dirigida al abasto de la industria y el mercado, sin poner atención en la complejidad de los ecosistemas forestales (Puettmann, Coates y Messier, 2009), ha desviado la atención del papel que juega la conservación como parte del manejo forestal, lo cual ha tenido consecuencias no solo en la degradación de las áreas forestales, sino también en la disminución de su productividad (Perry 1998).

No obstante, algo muy importante a considerar es la resiliencia, que constituye una característica de los bosques referida a su capacidad de recuperarse tras fenómenos de perturbación importantes (Gunderson, 2000). Bajo la mayor parte de los regímenes de perturbación natural, los 
bosques consiguen mantener su resiliencia en el tiempo. La resiliencia forestal es una propiedad ecosistémica emergente que deriva de la biodiversidad en múltiples escalas, y comprende desde la diversidad genética hasta la diversidad paisajística (Thompson, Mackey, McNulty y Mosseler, 2009).

En general, según Tittler, Filotas, Kroese y Messier (2015) y Vergara, Palma y Sepúlveda (2015), la gestión sostenible de los recursos naturales involucra decidir la estrategia más adecuada para su aprovechamiento y cuidado. En particular, gestionar el aprovechamiento y cultivo de los bosques implica decidir cuándo y dónde cosechar para satisfacer la demanda de productos forestales y al mismo tiempo cumplir con las políticas sociales de conservación; implica encontrar estrategias de equilibrio entre las demandas de la industria forestal y otros valores ecológicos de interés para la sociedad. Bouchard, D’Amours, Rönnqvist, Azouzi y Gunn (2017) afirman, por su parte, que en el pasado las estrategias de aprovechamiento de los bosques se enfocaban a maximizar el ingreso en un horizonte de planeación. Actualmente, uno de los objetivos de la gestión forestal es la conservación del ecosistema, además de la provisión de otros servicios ecosistémicos.

\section{CONCLUSIONES}

Sesenta porciento de las concesiones forestales (CF1, CF2 y CF3) cuyo aprovechamiento forestal se realiza de manera manual, son sustentables ambientalmente, evidenciando que este tipo de aprovechamiento forestal mantiene armonía con el entorno natural.

Existe fuerte correlación positiva estadísticamente significativa entre los indicadores "conservación y mantenimiento del recurso suelo" (CMRS) y "conservación de flora silvestre" (CFIS); es decir, a mejores y mayores acciones de conservación y mantenimiento del recurso suelo, mayores posibilidades de conservar la flora silvestre, $\mathrm{y}$ viceversa.

La concesión forestal CF1 cuenta con un solo manantial sin contaminación orgánica e inorgánica, por lo que el indicador "agua para consumo humano directo"
(APCHD) obtuvo un valor 1.0; y la concesión CF3 alcanzó un valor 1.0 para "aire libre de contaminación" (ALC), debido a que, según el titular de la concesión forestal, el aire se encuentra libre de polvo y residuos agroquímicos, en tanto el valor 0 de "conservación de fauna silvestre" (CFaS), muestra solo dos evidencias de la presencia de fauna silvestre: colpas y bañaderos.

Las concesiones forestales CF4 y CF5 que realizan aprovechamiento forestal mecanizado, resultaron no sustentables ambientalmente (índice de sustentabilidad ambiental menor a 2.0).

Pese a que la concesión forestal CF4 realiza aprovechamiento forestal mecanizado de impacto reducido, el valor 0 del indicador "agua para consumo humano directo" (APCHD) evidencia que no tiene ningún manantial libre de contaminación orgánica e inorgánica; el valor 1 para "conservación de flora silvestre" (CFIS) muestra la ocurrencia de cuatro incendios forestales en los últimos 15 años; y el valor 1.0 para "conservación de fauna silvestre" ( $\mathrm{CFaS})$, revela tres evidencias de la presencia de fauna silvestre: madrigueras, nidos de aves y bebederos.

La concesión forestal CF5 presenta valor 0 para el indicador "aire libre de contaminación" (ALC), refiriendo que solo está libre de polvo como agente contaminante; el valor 0.20 para "conservación y mantenimiento del recurso suelo" (CMRS), evidencia una mayor compactación debido al aprovechamiento forestal mecanizado, y que la diversidad de macrofauna del suelo según ShannonWienner es menor igual a 1.30 a una profundidad de $0 \mathrm{~cm}$ y $30 \mathrm{~cm}$; y el valor 0 de "conservación de flora silvestre" (CFIS) advierte cinco incendios en los últimos 15 años.

\section{REFERENCIAS}

Akay, A., Yilmaz, M., \& Tongue, F. (2006). Impact of mechanized harvesting machines on forest ecosystem: Residual stand damage. Journal of Applied Sciences, 6(11), 2414-2419. doi: $\underline{10.3923 / \text { jas.2006.2414. } 2419}$

Anderson, J., \& Ingram, J. (1993). Tropical soil biology and fertility: A handbook of methods. Soil Science, 157(4), 265. doi: $10.2307 / 2261129$ 
Bierbaß, P., Wündsch, M., \& Michalzik, B. (2014). The impact of vegetation on the stability of dispersive material forming biancane badlands in Val d'Orcia, Tuscany, Central Italy. Catena, 113, 260266. doi: $10.1016 /$ i.catena.2013.08.003

Bouchard, M., D'Amours, S. Rönnqvist, M., Azouzi, R., \& Gunn, E. (2017). Integrated optimization of strategic and tactical planning decisions in forestry. European Journal of Operational Research, 259(3), 1132-1143. doi: 10.1016/j.ejor.2016.11.022

Cano, P. A., Martínez, O. U., Martínez, M., \& Quiñones, A. (2007). Criterios e indicadores para evaluar el uso y manejo sustentable de bosques templados en Coahuila. Inifap-Cirne. Campo Experimental Saltillo. Publicación especial, 12, 45.

Carabelli, E., \& Peri, P. L. (2005). Criterios e indicadores de sustentabilidad (C\&̈I) para el manejo sostenible de los bosques nativos de Tierra del Fuego, Argentina: Una herramienta metodológica para la determinación de los C\&I en Patagonia (1a ed.). Río Gallegos, Santa Cruz, Argentina: INTA.

Chen, J., Franklin, J. F., \& Spies, T. A. (1995). Growing-season microclimatic gradients from clearcut edges into old-growth Douglas-fir forests. Ecological Applications, 5, 74-86. doi: 10.2307/ 1942053.

Consultora Internacional Independiente [Indufor]. (2012). Plan de Inversión Forestal. Componente III: elementos para la identificación de áreas con mayor potencial para reducir emisiones de GEI en el sector forestal. Lima, Perú: Indufor.

Contreras, F., Cordero, W., \& Fredericksen, T. S. (2001). Evaluación del aprovechamiento forestal. Cobija, Bolivia: Proyecto de Manejo Forestal Sostenible (Bolfor).

Cruz, M. (2003). Evaluación de un aprovechamiento forestal en bosque latifoliado y elaboración de tablas de volumen en la zona atlántica de Honduras. La Ceiba, Honduras: Administración Forestal del Estado (AFECohdefor)-Organización Internacional de Maderas Tropicales (OIMT).

Dezzeo, N., Chacón, N., Sanoja, E., \& Picón, G. (2004). Changes in soil properties and vegetation characteristics along a forest-savanna gradient in southern Venezuela. Forest Ecology and Management, 200(1-3), 183-193. doi: 10.1016/i.foreco.2004.06.016

Floyd, D. W., Vonhof, S. L., Seyfang, H. E., Heissenbuttel, J., Cantrell, R., Stocker, L., Wilkinson, B., \& Connaughton, K. (2001). Forest sustainability: A discussion guide for profesional resource managers, Journal of Forestry, 99(2), 8-28. doi: 10.1093/jof/ 99.2.8

Fredericksen, T. S. (2000). Logging and conservation of tropical forests in Bolivia. International Forestry Review, 2(4), 271-278.

Gayoso, J., \& Iroumé, A. (1991). Metodología para estimar la fragilidad de terrenos forestales. Medio Ambiente (V aldivia), 11(2), 13-24.
Gobierno Regional de Huánuco [Gorehco]. (2016). Zonificación ecológica y económica: estudio climático provincia de Leoncio Prado. Huánuco, Perú: Gorehco.

Gobierno Regional de Huánuco [Gorehco]. (2016a). Zonificación ecológica y económica: estudio climático provincia de Marañón. Huánuco, Perú: Gorehco.

Gobierno Regional de Huánuco [Gorehco]. (2016b). Zonificación ecológica y económica: estudio climático provincia de Puerto Inca. Huánuco, Perú: Gorehco.

Grossberg, S. P. (2009). Forest management. New York, United States: Nova Science Publishers, Inc.

Gunderson, L. (2000). Ecological resilience: In theory and application. Annual Review of Ecology, Evolution and Systematics, 31, 425-439. doi: 10.1146/ annurev.ecolsys.31.1.425

Hammond, A., Adriaanse, A., Rodenburg, E., Bryant, D., \& Woodward, R. (1995). Environmental indicators: A systematic approach to measuring and reporting on environmental policy performance in the context of sustainable development. Washington, D.C., Estados Unidos: World Resources Institute.

Hawksworth, D. L., \& Bull, A. T. (2006). Forest diversity and management. Dordrecht, Netherlands: Springer.

Hernández, R. (2014). Metodología de la investigación (6 $6^{\mathrm{a}}$ ed.). México D.F., México: McGraw-Hill/Interamericana Editores, S.A.

Hens, L., \& De Wit, J. (2003). The development of indicators and core indicators for sustainable development: A state of the art review. International Journal of Sustainable Development, 6(4), 436-459. doi: $\underline{10.1504 / I J S D .2003 .004188}$

Herrera-Ulloa, Á., Lluch-Cota, S., Ramírez-Aguirre, H., HernándezVázquez, S., \& Ortega-Rubio, A. (2003). Sustainable performance of the tourist industry in the state of Baja California Sur, México. Interciencia, 28(5), 268-272. issn: 0378-1844

Hildebrand, E. E., \& Schack-Kirchner, H. (2002). The influence of compaction on soil structure and functions in forest sites. En R. S. Ambasht, \& N. K. Ambasht (Eds.), Modern trends in applied terrestrial ecology (pp. 1-11). New York, Estados Unidos: Kluwer Academic Publihers.

Lefroy, R., Bechstedt, H., \& Rais, M. (2000). Indicators for sustainable land management based on farmer surveys in Vietnam, Indonesia, and Thailand. Agriculture, Ecosystems \& Environment, 81(2), 137146. doi: 10.1016/S0167-8809(00)00187-0

Ley $N^{\circ} 29763$ Ley Forestal y de Fauna Silvestre. Diario Oficial El Peruano, Lima, Perú, 22 de julio de 2011.

Lozada, J., \& Arends, E. (1998). Impactos ambientales del aprovechamiento forestal en Venezuela. Interciencia, 23(2), 74-83. 
Mackensen, J., Tillery-Stevens, M., Klinge, R., \& Fölster, H. (2000). Site parameters, species composition, phytomass structure and element stores of a terra-firme forest in East-Amazonia, Brazil. Plant Ecology, 151(2), 101-119. doi: 10.1023/A:1026515116944

Masera, O., Astier, M., \& López-Ridaura, S. (1999). Sustentabilidad y manejo de recursos naturales: El marco de evaluación MESMIS. México D.F.: Mundi-Prensa, GIRA e Instituto de Ecología UNAM.

Meza, C., \& Díaz, A., I. (2010). Percepción ambiental de los paisajes y sus potencialidades: provincia de Huamalíes. Investigaciones Sociales, 14(25), 47-62. doi: 10.15381/is.v14i25.7293

Morse, S., McNamara, N., \& Acholo, M. (2004). Soils, souls and agricultural sustainability: The need for connection. International Journal of Sustainable Development, 7(4), 410-432. doi: 10.1504/ IJSD.2004.006417

Narváez, R., Wright, P., Martínez, M., Alvídrez, S., Domínguez, L., Gómez, V., Rodríguez, S., Montes, G., Molina, J., \& Iglesias, L. (2003). Criterios e Indicadores, una berramienta para evaluar la sustentabilidad del manejo forestal en bosques templados y tropicales ( $1^{\mathrm{a}} \mathrm{ed}$.) México: Instituto Nacional de Investigaciones Forestales, Agrícolas y Pecuarias.

Navarro, J., de Araújo, J. C., \& Mongil, J. (2014). Assessment of 80 years of ancient-badlands restoration in Saldaña, Spain. Earth Surface Processes and Landforms, 39(12), 1563-1575. doi: 10.1002/esp.3541

Neary, D. G., Ice, G. G., \& Jackson, C. R. (2009). Linkages between forest soils and water quality and quantity. Forest Ecology and Management, 258(10): 2269-2281. doi: $\underline{10.1016 / \text { i.foreco. } 2009.05 .027}$

Organización de las Naciones Unidas para la Alimentación y la Agricultura [FAO]. (2003). Trade and sustainable forest management impacts and interactions. Roma, Itallia: Forest Products Division.

Orozco, L., Brumér, C., \& Quirós, D. (2006). Aprovechamiento de impacto reducido en bosques latifoliados búmedos tropicales. Turrialba, Costa Rica: Centro Agronómico Tropical de Investigación y Enseñanza (Catie).

Padmawathe R, Qureshi Q, \& Rawat G. S. (2004). Effects of selective logging on vascular epiphyte diversity in a moist lowland forest of Eastern Himalaya, India. Biological Conservation, 119(1), 81-92. doi: 10.1016/j.biocon.2003.10.024

Pearson, K. (1920). Notes on the history of correlation. Biometrika, 13(1), 25-45. doi: 10.1093/biomet/13.1.25

Perry, D. A. (1998). The scientific basis of forestry. Annual Review of Ecology and Systematics 29(1), 435-466. doi: $\underline{10.1146 / \text { annurev.ecolsys.29.1.435 }}$
Pokorny, B., Sabogal, C., \& de Camino, R. (2001). Metodologías para evaluar la aplicación de criterios e indicadores en el manejo forestal de bosques tropicales en América Latina. Revista Forestal Centroamericana, 36, 14-19.

Prabhu, R., Colfer, C. J. P., Venkaterswarlu, P., Tan, L. C., Soekmadi, R., \& Wollenberg, E. (1996). Testing criteria and indicators for the sustainable management of forests: phase 1. Final Report. Jakarta, Indonesia: Center for International Forestry Research (CIFOR).

Puettmann, K. J., Coates, K. D., \& Messier, Ch. (2009). A critique of silviculture. Managing for complexity. Washington D.C., United States of America: Island Press.

Sarandón, S. (2002). El desarrollo y uso de indicadores para evaluar la sustentabilidad de los agroecosistemas. En S. J. Sarandón (Ed.), Agroecología: El camino hacia una agricultura sustentable (pp. 393-414). La Plata, Argentina: Ediciones Científicas Americanas.

Sarandón, S., \& Flores, C. (2009). Evaluación de la sustentabilidad en agroecosistemas: Una propuesta metodológica. Agroecología, 4, 1928.

Smith W. (2002). Developing Indicators of 'sustainability'. En I.R. Bowler, C.R. Bryant, \& C. Cocklin (Eds.), The sustainability of rural systems: Geographical interpretations (pp. 13-34). Dordrecht, The Netherlands: Kluwer Academic Publishers.

Solgi, A., \& Najafi, A. (2007). Investigating of residual tree damage during ground-based skidding. Pakistan Journal of Biological Sciences, 10, 1755-1758. doi: $10.3923 /$ pjbs.2007.1755.1758

Thompson, I., Mackey, B., McNulty, S., \& Mosseler, A. (2009). Forest resilience, biodiversity, and climate change. A synthesis of the biodiversity/resilience/ stability relationship in forest ecosystems. Montreal, Quebec, Canadá: Secretariat of the Convention on Biological Diversity.

Toledo, V. (1998). Estudiar lo rural desde una perspectiva interdisciplinaria: el enfoque ecológico-sociológico. En Globalización, crisis y desarrollo rural en América Latina. Memoria de sesiones plenarias del V Congreso Latinoamericano de Sociología Rural (pp. 159-179). Texcoco, México: Asociación Latinoamericana de Sociología Rural (Alasru), Universidad Autónoma de Chapingo, Colegio de Posgraduados.

Thornthwaite, C. W. (1948). An approach toward a rational classification of climate. Geographical Review, 38(1), 55-94. doi: 10.2307/210739

Tittler, R., Filotas, É., Kroese, J., \& Messier, Ch. (2015). Maximizing conservation and production with intensive forest management: It's all about location. Environmental Management, 56(5), 1104 1117. doi: 10.1007/s00267-015-0556-3

Torri, D., Santi, E., Marignani, M., Rossi, M., Borselli, L., \& Maccherini, S. (2013). The recurring cycles of biancana badlands: Erosion, 
vegetation and human impact. Catena, 106, 22-30. doi: 10.1016/j.catena.2012.07.001

United States Department of Agriculture [Usda]. (2002). Sourcebook on criteria and indicators of forest sustainability in the Northeastern Area. Newtown Square, Philadelphia, Pennsylvania, EE.UU.: USDA Forest Service Northeastern Area State and Private Forestry.

Valentin, C., Poesen, J., \& Li, Y. (2005). Gully erosion: Impacts, factors and control. Catena, 63(2-3 Special Iss.), 132-153. doi: $\underline{10.1016 / \text { i.catena.2005.06.001 }}$

Velarde S. J., Ugarte-Guerra, J., Tito, M. R., Capella, J. L., Sandoval, M., Hyman, G., Castro, A., Marín, J. A., \& Barona, E. (2010). Reducing emissions from all land uses in Peru. Final national report. Nairobi, Kenya: ASB Partnership for the Tropical Forest Margins.

Vergara, F. P., Palma, C. D., \& Sepúlveda, H. (2015). A comparison of optimization models for lumber production planning. Bosque (V aldivia), 36(2), 239-246. 이 $\quad 10.4067 /$ S0717$\underline{92002015000200009}$

World Commission on Environment and Development. (1987). Our common future. New York, Estados Unidos: Oxford University Press.

Zhang, Y., Zhou, Z., Cheng, J., \& Zhang, H. (2013). Soil quality and plant diversity in managed forest in the hilly purple soil, southwestern China. Journal of Food, Agriculture and Environment, 11(1), 945-951.
Zwane, A. P. 2007. Does poverty constrain deforestation? Econometric evidence from Peru. Journal of Development Economics, 84(1), 330349. doi: $10.1016 /$ i.jeveco.2005.11.007

Manuscrito recibido el 24 de octubre de 2019

Aceptado el 27 de marzo de 2020

Publicado el 04 de diciembre de 2020

Este documento se debe citar como:

Valdivia E., L. A., Gonzalez M. L., T. F., \& Julca-Otiniano, A. M. (2020). Sustentabilidad ambiental de las concesiones forestales en el departamento Huánuco, Perú. Madera y Bosques, 26(3), e2632062. doi: 10.21829/myb.2020.2632062

Madera y Bosques por Instituto de Ecología, A.C. se distribuye bajo una Licencia Creative Commons Atribución-NoComercialCompartirlgual 4.0 Internacional. 